\title{
HIDROCARBONETOS POLICÍCLICOS AROMÁTICOS (HPAS) EM CACHAÇA, RUM, UÍSQUE E ÁLCOOL COMBUSTÍVEL
}

\author{
Carlos Alexandre Galinaro e Douglas Wagner Franco* \\ Instituto de Química de São Carlos, Universidade de São Paulo, CP 780, 13560-970 São Carlos - SP, Brasil
}

Recebido em 20/6/08; aceito em 23/1/09; publicado na web em 3/7/09

\begin{abstract}
POLYCYCLIC AROMATIC HYDROCARBONS (PAHs) IN CACHAÇA, RUM, WHISKEY AND ALCOHOL FUEL. The concentration of 15 polycyclic aromatic hydrocarbons (PAHs) in 57 samples of distillates (cachaça, rum, whiskey, and alcohol fuel) has been determined by HPLC-Fluorescence detection. The quantitative analytical profile of PAHs treated by Partial Least Square - Discriminant Analysis (PLS-DA) provided a good classification of the studied spirits based on their PAHs content. Additionally, the classification of the sugar cane derivatives according to the harvest practice was obtained treating the analytical data by Linear Discriminant Analysis (LDA), using naphthalene, acenaphthene, fluorene, phenanthrene, anthracene, fluoranthene, pyrene, benz[a] anthracene, benz[b]fluoranthene, and benz[g,h,i]perylene, as a chemical descriptors.
\end{abstract}

Keywords: PAHs; beverages; alcohol fuel.

\section{INTRODUÇÃo}

Hidrocarbonetos policíclicos aromáticos (HPAs) são substâncias que pertencem à classe dos pró-carcinogênicos, isto é, necessitam sofrer ativação metabólica preliminar para formar o carcinógeno ativo e tornarem-se capazes de reagir com o DNA e outras moléculas. ${ }^{1-4}$ São formados pela queima de matéria orgânica em elevadas temperaturas, por meio da combustão incompleta ou pirólise de materiais contendo átomos de carbono e hidrogênio..$^{5-7}$

A presença dos HPAs em alimentos pode decorrer dos processos de defumação, torrefação, secagem direta com madeira, poluição do solo e da água. ${ }^{6,7}$ Em derivados de cana-de-açúcar e bebidas destiladas, a formação de hidrocarbonetos pode ocorrer durante as etapas de produção. ${ }^{6-8}$

A cachaça e o rum podem sofrer a contaminação por HPAs pela queima da cana-de-açúcar durante a colheita, ${ }^{9,10}$ por meio de adição de caramelo (utilizado para a correção da coloração da bebida envelhecida) no produto final ou durante o período de maturação (envelhecimento $)^{8,9} \mathrm{em}$ tonéis de madeira cuja parte interior foi submetida à queima durante sua confecção. ${ }^{11,12}$

O álcool combustível (álcool etílico hidratado combustível) é outro derivado da cana, cuja utilização como combustível e matéria prima para a indústria está sendo altamente estimulado por ser proveniente de fonte renovável, além de ser econômico e ambientalmente vantajoso, ${ }^{3}$ quando comparado com derivados de petróleo. Assim como verificado para outros derivados de cana-de-açúcar, ${ }^{9,10}$ a presença de hidrocarbonetos no etanol estaria associada ao processo de colheita da cana utilizada na sua produção.

Em bebidas preparadas a partir do malte, como o uísque escocês, a contaminação pode ocorrer durante a secagem dos maltes germinados da cevada pelo processo de defumação e durante a estocagem em barris de madeira, ${ }^{8}$ cujo interior foi submetido ao processo de queima. Para os uísques de origem americana, a presença dos HPAs também pode vir a ocorrer com a passagem destas bebidas em filtros de carvão vegetal, ${ }^{8}$ utilizados para a retirada de compostos indesejáveis.

Procurando contribuir para o melhor conhecimento do tema, o presente trabalho relata os teores médios de 15 HPAs determinados em amostras de cachaça, rum, uísque e álcool combustível. O tratamento

*e-mail: douglas@iqsc.usp.br destes resultados por meio de análise quimiométrica (Partial Least Square-Discriminant Analisys, PLS-DA $)^{13,14}$ objetivando verificar eventuais similaridades entre o perfil de HPAs presentes nas amostras de cachaça, rum e uísque também é apresentado. Foi aplicado aos resultados das amostras de derivados de cana analisadas (cachaça, rum e álcool combustível) um modelo quimiométrico ${ }^{15}$ desenvolvido anteriormente, utilizando-se a técnica de análise discriminante linear (Linear Discriminant Analysis, LDA) para reconhecer o tipo de cana (queimada ou não queimada) utilizado na sua produção.

\section{PARTE EXPERIMENTAL}

\section{Amostras}

Foram analisadas 14 amostras de cachaça comerciais, 19 de rum, 18 de uísque e 6 de álcool combustível. As relações das amostras analisadas, bem como a sua origem, encontram-se descritas a seguir.

As cachaças, adquiridas diretamente dos produtores, e por estes certificadas foram: Água Branca (Brasil, Brotas, SP), Armazém Vieira Esmeralda (Brasil, Florianópolis, SC), Armazém Vieira Onix (Brasil, Florianópolis, SC), Armazém Vieira Rubi (Brasil, Florianópolis, SC), Armazém Vieira Tradicional (Brasil, Florianópolis, SC), Beija-Flor (Brasil, Gruarapiranga, SP), Brotas (Brasil, Brotas, SP), Cafelândia (Brasil, Cafelândia, SP), Gerbi (Brasil, Mogi Mirim, SP), Grizzo (Brasil, Jaú, SP), Lucato (Brasil, Pontal, SP), Santo Mário (Brasil, Catanduva, SP), São Bento (Brasil, Batatais, SP) e São João (Brasil, Torrinha, SP).

O rum e os uísques, adquiridos em lojas de Free Shopping, foram. Rum: Appleton Estate (Jamaica), Bacardi Carta de Oro (Brasil), Bacardi Carta Blanca (Brasil), Bucanero Añejo (Cuba), Cacique Premium Silver (Venezuela), Capitain Morgan Dark (Canadá), Conde de Cuba Añejo 7 anos (Cuba), Cortez Añejo 3 anos (Panamá), Cruzan Estate Diamond (Ilhas Virgens), El Dorado 12 years (Guiana), Flor de Cana Black Label (Nicarágua), Havana Club Añejo 3 anos (Cuba), Havana Club Añejo 7 Anos (Cuba), Havana Club Añejo Reserva (Cuba), Havana Club Silver Dry (Cuba), Santiago de Cuba Añejo (Cuba), Solera XK (México), Varadero Añejo 7 anos (Cuba) e Vigia Gran Añejo (Cuba). Uísques: Ballantines (Escócia), Blend Seven 7 Crown (EUA), Buchanans (Escócia), Evan Willians (EUA), Four Roses (EUA), Glenfiddich (Escócia), Grants (Escócia), Jack Daniel's (EUA), Jameson (Irlanda), Jim Beam 
(EUA), Johnnie Walker Black Label (Escócia), Johnnie Walker Blue Label (Escócia), Johnnie Walker Gold Label (Escócia), Markers Mark (EUA), Old Grand Dad (EUA), Passport Scotch (Escócia), Tullamore Dew (Irlanda) e Wild Turkey (EUA).

As amostras de álcool combustível foram adquiridas em postos de combustível: Esso ${ }^{\circledR}$ (Brasil, SP, Cajuru), Ipiranga ${ }^{\circledR}$ (Brasil, SP, São Carlos), Petrobrás ${ }^{\circledR}$ (Brasil, SP, São Carlos), Shell ${ }^{\circledast}$ (Brasil, SP, Cajuru), Shell ${ }^{\circledR}$ (Brasil, SP, São Carlos), Texaco ${ }^{\circledR}$ (Brasil, SP, Ribeirão Preto).

\section{Materiais}

Os padrões utilizados para a análise dos HPAs foram todos de pureza analítica adquiridos da Sigma-Aldrich (Steinheim, Germany) e estão listados a seguir: naftaleno (NA), acenafteno (AC), fluoreno (F), fenantreno (FE), antraceno (AN), fluoranteno (FL), pireno (PI), benzo[a] antraceno $(\mathrm{BaA})$, criseno $(\mathrm{CR})$, benzo[b]fluoranteno $(\mathrm{BbF})$, benzo[k]fluoranteno $(\mathrm{BkF})$, benzo[a]pireno $(\mathrm{BaP})$, dibenzo[a,h]antraceno (DBahA), benzo[g,h,i]perileno (BghiP) e indeno[1,2,3-c,d]pireno (IP).

A partir de uma solução estoque de HPAs $0,100 \mathrm{mg} \mathrm{L}^{-1}$ em acetonitrila/metanol (1:1 v/v), foram preparadas em acetonitrila soluções padrão com concentrações que variaram de 50 a $500 \mu \mathrm{g} \mathrm{L}{ }^{-1}$.

\section{Extração}

Em função dos teores de HPAs em destilados serem da ordem de $n g \mathrm{~L}^{-1}$ a $\mu \mathrm{g} \mathrm{L}^{-1}$, as amostras foram submetidas a uma etapa de concentração e limpeza (clean-up) por extração em fase sólida (solid phase extraction, SPE), para a retirada de possíveis componentes que pudessem interferir nas análises e comprometer a vida útil da coluna, seguindo-se metodologia existente na literatura. ${ }^{9}$ As amostras de álcool combustível, que apresentam teor alcoólico de 92,6-92,8 ${ }^{\circ} \mathrm{GL}$, foram diluídas com água MILI-Q (1:1) visando-se aproximar o teor alcoólico do combustível ao das bebidas analisadas (38 a 54\% v/v).

\section{Equipamentos}

Os HPAs foram analisados utilizando-se um cromatógrafo líquido (Shimadzu), modelo LC-10AD, equipado com injetor (Shimadzu) com loop de $5 \mu \mathrm{L}$, acoplado a um detector de fluorescência, modelo RF-551 (Shimadzu). A coluna utilizada foi de fase reversa C18 Supelcosil LC-PAH (Supelco, Bellefonte, PA), de $25 \mathrm{~cm} \mathrm{x} \mathrm{4,6}$ $\mathrm{mm}$ d.i., com partículas de $5 \mu \mathrm{m}$ de tamanho, acoplada a uma précoluna GVP-ODS (Shimadzu), de 5 L x 2,0 d.i.. As amostras foram sempre injetadas em triplicata e quantificadas por adição de padrão. A metodologia utilizada para a análise de HPAs em destilados foi previamente descrita na literatura. ${ }^{9}$

\section{Análise quimiométrica}

O banco de dados das análises quantitativas das amostras de cachaça, rum e uísque da $P L S$-DA foi auto escalonado e organizado na forma de uma matriz 15 x 51 para a $P L S$ - $D A .{ }^{16}$ Já na $L D A$, utilizou-se uma matriz 15 x 39 para as amostras de cachaça, rum e álcool combustível. A análises dos resultados experimentais, segundo as técnicas quimiométricas ${ }^{13,16,17}$ de $P L S$-DA e $L D A$, foi realizada utilizando-se o programa MINITAB Release 14 (Statistical Software, State College, Pennsylvania, EUA).

\section{RESULTADOS E DISCUSSÃO}

A Figura 1 ilustra os cromatogramas típicos obtidos para amostras de cachaça (A), rum (B), uísque (C), álcool combustível (D) e da mistura dos padrões de HPAs (E). Nota-se que a metodologia ${ }^{9}$
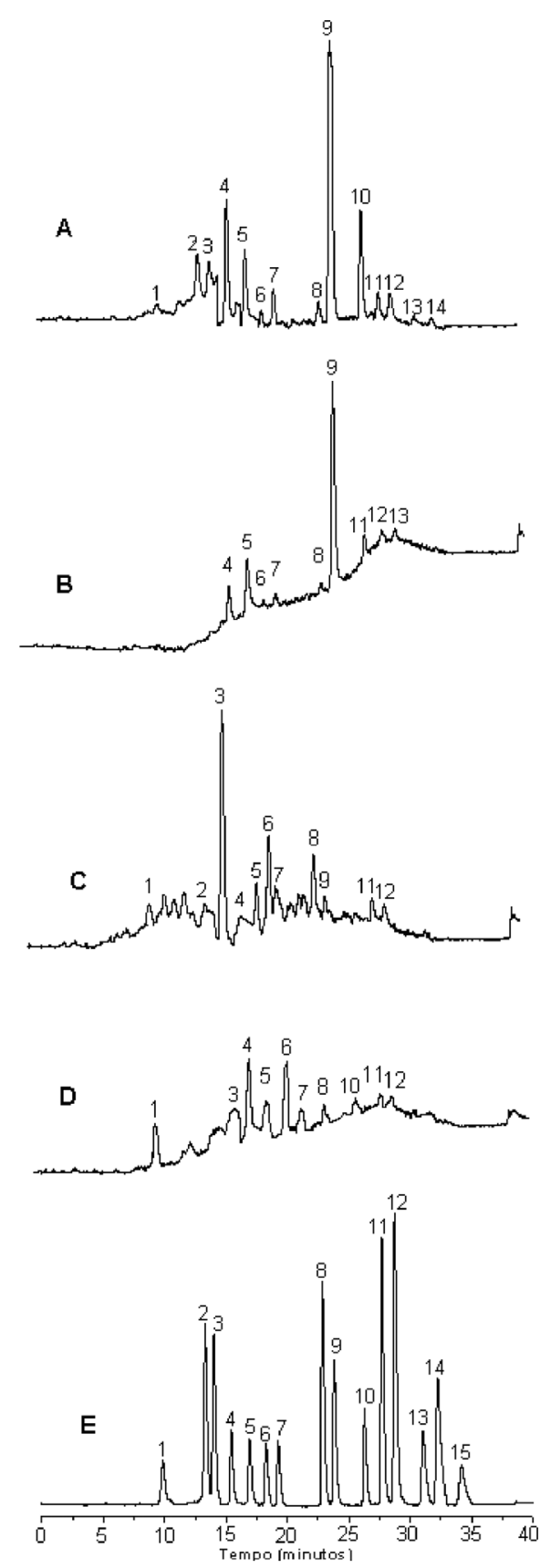

Figura 1. Cromatogramas típicos: $A=$ cachaça, $B=$ rum, $C=$ uísque, $D=$ álcool combustível, $E=$ mistura de $25 \mu \mathrm{g} L^{-1}$ dos padrões de HPAs. $1=N A$, $2=A C, 3=F, 4=F E, 5=A N, 6=F L, 7=P I, 8=B a A, 9=C R, 10=B b F$ $=11=B k F, 12=B a P, 13=D B a h A, 14=B g h i P, 15=I P$

conduz a uma boa separação para todos os 15 HPAs estudados, com um tempo total de análise de aproximadamente $36 \mathrm{~min}$.

As características da metodologia (índice de recuperação, desvio padrão relativo, repetibilidade, reprodutibilidade, linearidade, limite de detecção e quantificação) utilizadas para a análise dos HPAs nos destilados estão apresentadas em trabalho anterior. ${ }^{9}$

A Tabela 1 apresenta a média, a mediana e a soma dos teores médios dos HPAs calculados com base nos resultados experimentais para as amostras de cachaça, rum, uísque e álcool combustível, respectivamente. Os resultados das concentrações de HPAs para todas as amostras analisadas encontram-se na Tabela 1S (Material Suplementar). Dos 15 HPAs analisados, 14 foram detectados nas 
Tabela 1. Média, mediana e soma dos teores médios dos HPAs $\left(\mu \mathrm{g} \mathrm{L}^{-1}\right)$ nas amostras de cachaça, rum, uísque e álcool combustível

\begin{tabular}{|c|c|c|c|c|c|c|c|c|}
\hline \multirow[b]{2}{*}{ HPAs $^{\mathrm{a}, \mathrm{b}}$} & \multicolumn{2}{|c|}{ Cachaça } & \multicolumn{2}{|c|}{ Rum } & \multicolumn{2}{|c|}{ Uísque } & \multicolumn{2}{|c|}{ Álcool Combustível } \\
\hline & Média & Mediana & Média & Mediana & Média & Mediana & Média & Mediana \\
\hline NA & 4,32 & 0,392 & 0,479 & 0,502 & 1,90 & 0,531 & 0,292 & 0,233 \\
\hline $\mathrm{AC}$ & 0,422 & 0,297 & 0,172 & $8,17 \times 10^{-2}$ & 0,620 & 0,152 & 0,182 & $9,39 \times 10^{-2}$ \\
\hline $\mathrm{F}$ & 0,386 & 0,248 & $4,28 \times 10^{-2}$ & $5,16 \times 10^{-2}$ & 0,592 & 0,122 & 0,386 & - \\
\hline $\mathrm{FE}$ & 1,26 & 0,905 & 0,333 & 0,191 & 1,10 & 0,497 & 0,834 & 0,140 \\
\hline $\mathrm{AN}$ & 0,454 & 0,324 & 0,165 & 0,109 & 0,236 & 0,436 & 0,988 & - \\
\hline FL & 0,667 & 0,519 & 0,233 & 0,164 & 0,910 & 0,281 & 0,972 & 0,750 \\
\hline PI & 0,526 & 0,565 & $7,14 \times 10^{-2}$ & $6,00 \times 10^{-2}$ & 0,101 & 0,329 & 0,597 & $7,73 \times 10^{-2}$ \\
\hline $\mathrm{BaA}$ & $9,83 \times 10^{-2}$ & $7,25 \times 10^{-2}$ & 0,259 & $9,70 \times 10^{-2}$ & 0,376 & $8,24 \times 10^{-2}$ & 0,185 & $6,02 \times 10^{-2}$ \\
\hline $\mathrm{CR}$ & 0,728 & 0,147 & 0,116 & $1,43 \times 10^{-2}$ & 0,646 & $9,64 \times 10^{-2}$ & 0,551 & $6,25 \times 10^{-2}$ \\
\hline $\mathrm{BbF}$ & 0,212 & $2,35 \times 10^{-2}$ & - & - & 0,107 & $5,52 \times 10^{-2}$ & 0,396 & - \\
\hline $\mathrm{BkF}$ & $2,52 \times 10^{-2}$ & $2,70 \times 10^{-2}$ & $4,51 \times 10^{-2}$ & $3,43 \times 10^{-2}$ & $8,36 \times 10^{-2}$ & $1,76 \times 10^{-2}$ & $1,70 \times 10^{-2}$ & $3,59 \times 10^{-3}$ \\
\hline $\mathrm{BaP}$ & $3,89 \times 10^{-2}$ & $3,65 \times 10^{-2}$ & $7,02 \times 10^{-3}$ & $5,31 \times 10^{-3}$ & $3,23 \times 10^{-2}$ & $1,15 \times 10^{-2}$ & $1,58 \times 10^{-2}$ & $8,25 \times 10^{-3}$ \\
\hline DBahA & $8,97 \times 10^{-2}$ & 0,101 & - & - & $4,99 \times 10^{-2}$ & $3,74 \times 10^{-2}$ & $2,99 \times 10^{-2}$ & \\
\hline BghiP & 0,203 & 0,171 & - & - & 0,124 & $9,28 \times 10^{-2}$ & 0,256 & $8,51 \times 10^{-2}$ \\
\hline IP & - & - & - & - & - & - & - & - \\
\hline$\sum$ médias & 9,43 & & 1,98 & & 7,30 & & 5,70 & \\
\hline
\end{tabular}

aLimite de detecção $\left(\mu \mathrm{g} \mathrm{L}^{-1}\right): \mathrm{NA}=3,58 \times 10^{-3} ; \mathrm{AC}=3,93 \times 10^{-3} ; \mathrm{F}=2,93 \times 10^{-3} ; \mathrm{FE}=2,02 \times 10^{-3} ; \mathrm{AN}=4,20 \times 10^{-3} ; \mathrm{FL}=1,28 \times 10^{-2} ; \mathrm{PI}=$ $6,95 \times 10^{-3} ; \mathrm{BaA}=1,68 \times 10^{-3} ; \mathrm{CR}=2,24 \times 10^{-3} ; \mathrm{BbF}=5,68 \times 10^{-3} ; \mathrm{BkF}=6,56 \times 10^{-3} ; \mathrm{BaP}=5,92 \times 10^{-3} ; \mathrm{DBahA}=1,08 \times 10^{-3} ; \mathrm{BghiP}=3,22$ x $10^{-3} ; \mathrm{IP}=4,54 \times 10^{-2}$.

${ }^{\mathrm{b}}$ Limite de quantificação ( $\boldsymbol{\mu g} \mathbf{L}^{-1}$ ): $\mathrm{NA}=7,16 \times 10^{-1} ; \mathrm{AC}=1,25 \times 10^{-1} ; \mathrm{F}=4,74 \times 10^{-1} ; \mathrm{FE}=8,81 \times 10^{-1} ; \mathrm{AN}=1,36 \times 10^{-1} ; \mathrm{FL}=5,88 \times 10^{-1}$; $\mathrm{PI}=1,12 \times 10^{-1} ; \mathrm{BaA}=8,56 \times 10^{-1} ; \mathrm{CR}=3,98 \times 10^{-1} ; \mathrm{BbF}=5,22 \times 10^{-1} ; \mathrm{BkF}=5,61 \times 10^{-1} ; \mathrm{BaP}=9,30 \times 10^{-2} ; \mathrm{DBahA}=8,29 \times 10^{-1} ; \mathrm{BghiP}$ $=4,49 \times 10^{-1} ; \mathrm{IP}=7,43 \times 10^{-1}$.

amostras de cachaça, e os valores de suas concentrações médias variaram de 4,32 $\mu \mathrm{g} \mathrm{L}^{-1}(\mathrm{NA})$ a $2,52 \times 10^{-2} \mu \mathrm{g} \mathrm{L} \mathrm{L}^{-1}(\mathrm{BkF})$. Em trabalho semelhante, Tfouni et al. ${ }^{10}$ analisaram a presença de 5 HPAs (BaA, $\mathrm{BbF}, \mathrm{BkF}, \mathrm{BaP}$ e DBahA) em 25 amostras de cachaça e, de forma semelhante, observou-se que o $\mathrm{BkF}$ foi o hidrocarboneto que se apresentou em menor concentração $\left(0,01 \mu \mathrm{g} \mathrm{L}^{-1}\right)$, ao passo que o $\mathrm{BbF}$ foi o mais abundante $\left(0,83 \mu \mathrm{g} \mathrm{L}^{-1}\right)$. Conforme descrito por Galinaro et al. ${ }^{9}$ para 131 amostras de cachaças paulistas, o BkF também foi o menos abundante dos HPAs, quer nas cachaças produzidas com cana queimada $\left(2,05 \times 10^{-2} \mu \mathrm{g} \mathrm{L}^{-1}\right)$ quer nas produzidas com cana não queimada $\left(8,62 \times 10^{-3} \mu \mathrm{g} \mathrm{L}^{-1}\right)$.

Nas amostras de rum, foram identificados e quantificados 11 hidrocarbonetos (NA, AC, F, FE, AN, FL, PI, BaA, CR, BkF, BaP) com concentrações médias que variaram de $0,479 \mu \mathrm{g} \mathrm{L} \mathrm{L}^{-1}$ (NA) a 7,02 $\mathrm{x} 10^{-3} \mu \mathrm{g} \mathrm{L} \mathrm{L}^{-1}(\mathrm{BaP})$. Estes valores são inferiores aos observados para as amostras de cachaça (Tabela 1), coerente com o fato de em Cuba não ser hábito a queima da cana-de-açúcar durante a sua colheita. ${ }^{18}$ GarcíaFalcón et al. ${ }^{19}$ relataram a presença de 7 HPAs em duas amostras de rum, mas quantificaram apenas três hidrocarbonetos: $\mathrm{BbF}, \mathrm{BkF}$ e $\mathrm{BaP}$, com teores médios de 1,$35 ; 0,700$ e $0,700 \mathrm{ng} \mathrm{L}^{-1}$, respectivamente. Segundo trabalho recente, ${ }^{9}$ os valores das concentrações médias para 15 HPAs em quatro amostras de rum variaram de 4,92 x 10-3 (BaP) a $1,78 \times 10^{-1} \mu \mathrm{g} \mathrm{L}^{-1}(\mathrm{CR})$. Estes resultados em conjunto apontam para um baixo teor de HPAs em amostras de rum.

Nas amostras de álcool etílico, utilizado como combustível para veículos, foram identificados e quantificados 14 hidrocarbonetos (Tabela 1) cujos valores das concentrações médias variaram de 1,58 x $10^{-2}(\mathrm{BaP})$ a $0,988 \mu \mathrm{g} \mathrm{L}^{-1}(\mathrm{AN})$.

Para as amostras de uísque (Tabela 1), os teores médios de HPAs variaram de $3,23 \times 10^{-2}$ (BaP) a $1,90 \mu \mathrm{g} \mathrm{L}^{-1}$ (NA), valores estes de mesma ordem de grandeza dos reportados por Kleinjans et al. ${ }^{8}$ para 18 amostras de uísque. Neste trabalho os teores médios variaram de $1,30 \times 10^{-2} \mu \mathrm{g} \mathrm{L}^{-1}$ (hidrocarbonetos com 5 anéis aromáticos) a 8,66 x $10^{-2} \mu \mathrm{g} \mathrm{L} \mathrm{L}^{-1}$ (hidrocarbonetos com 3 anéis aromáticos).

Observa-se (Tabela 1) que as amostras de rum foram, dentre os destilados estudados, as que apresentaram a menor quantidade de hidrocarbonetos quantificados (11) e também a menor soma das concentrações médias $\left(1,98 \mu \mathrm{g} \mathrm{L}^{-1}\right)$ para os 15 HPAs analisados, seguidas pelas amostras de álcool combustível $\left(5,70 \mu \mathrm{g} \mathrm{L} \mathrm{L}^{-1}\right)$, uísque $\left(7,30 \mu \mathrm{g} \mathrm{L}^{-1}\right)$ e cachaça $\left(9,43 \mu \mathrm{g} \mathrm{L}^{-1}\right)$.

Até o presente momento não existe na legislação internacional limitação aceita universalmente para os teores de HPAs em água e alimentos. Na falta destes limites legais, alguns países passaram a adotar limites próprios. Por exemplo, na Alemanha, a German Society for Fat Science ${ }^{20,21}$ fixou para alimentos defumados o limite de $25 \mu \mathrm{g} \mathrm{L}^{-1}$ para os HPAs totais, e de $5 \mu \mathrm{g} \mathrm{L}^{-1}$ para os HPAs de alto peso molecular. Também na Europa, a Legislação da Comunidade Européia ${ }^{4}$ estabelece valores máximos de 1,0 a $10,0 \mu \mathrm{g} \mathrm{kg}^{-1}$ de BaP para alimentos (cereais, óleos, crustáceos, carnes defumadas, etc). No Brasil, a Legislação determina para águas envasadas ${ }^{22}$ teores de benzo(a)pireno inferiores a $0,7 \mu \mathrm{g}$ $\mathrm{L}^{-1}$. Já para alimentos aromatizados ou com aromas de fumaça, ${ }^{23}$ o limite de BaP é $0,03 \mu \mathrm{g} \mathrm{kg}{ }^{-1}$.

A Figura 2 apresenta a soma dos teores médios dos HPAs de baixo peso molecular (dois e três anéis aromáticos) e de alto peso molecular (quatro a seis anéis aromáticos) para as amostras de bebidas estudadas. Observa-se que a soma dos teores médios dos HPAs de baixo peso molecular (NA, AC, F, FE e AN) é maior que a soma dos HPAs de alto peso molecular (FL, PI, BaA, CR, BbF, $\mathrm{BkF}, \mathrm{BaP}, \mathrm{DBahA}$, BghiP e IP) para cachaça, rum e o uísque. 


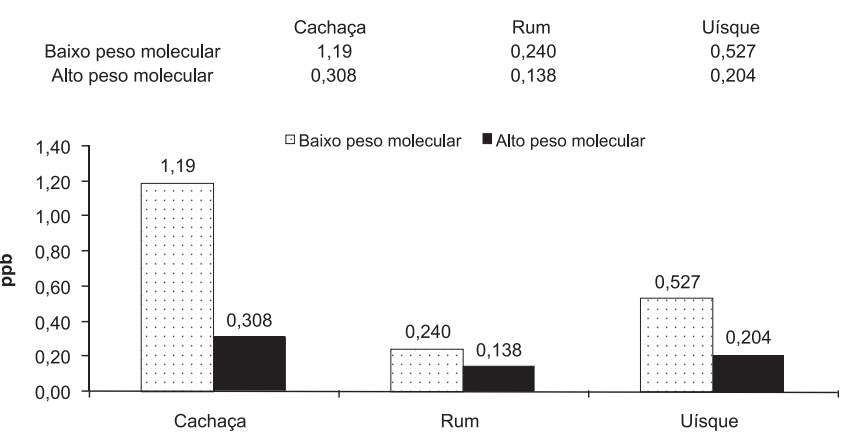

Figura 2. Soma dos teores médios dos HPAs $\left(\mu g L^{-1}=p p b\right)$ de baixo e alto peso molecular nas amostras de cachaça, rum e uísque

Da comparação entre os teores totais de HPAs sugeridos pela German Society for Fat Science, ${ }^{20,21}$ com os obtidos para as bebidas aqui mencionadas (Tabela 1), nota-se que a cachaça $\left(9,43 \mu \mathrm{g} \mathrm{L}^{-1}\right), \mathrm{o}$ rum $\left(1,98 \mu \mathrm{g} \mathrm{L}^{-1}\right)$ e o uísque $\left(7,30 \mu \mathrm{g} \mathrm{L}^{-1}\right)$ apresentam teores totais (soma) abaixo do limite estabelecido $\left(25 \mu \mathrm{g} \mathrm{L}^{-1}\right)$. Isto também ocorre quando se compara com o limite de $\mathrm{BaP}$ monitorado pela Legislação da Comunidade Européia ${ }^{4}\left(1,0\right.$ a $\left.10,0 \mu \mathrm{g} \mathrm{kg}^{-1}\right)$ e pela Legislação Brasileira $^{23}\left(0,03 \mu \mathrm{g} \mathrm{kg}^{-1}\right)$, ambas para alimentos: cachaça $(1,80 \mathrm{x}$ $\left.10^{-2} \mu \mathrm{g} \mathrm{kg}^{-1}\right)$, rum $\left(3,26 \times 10^{-3} \mu \mathrm{g} \mathrm{kg}{ }^{-1}\right)$ e uísque $\left(1,52 \times 10^{-2} \mu \mathrm{g} \mathrm{kg}^{-1}\right)$.

Com relação ao teor de $\mathrm{BaP}$ (Tabela 1S) estabelecido pela Legislação brasileira para águas envasadas, ${ }^{22}$ as amostras de bebidas estudadas (teor máximo) também estariam de acordo com o limite permitido de $0,7 \mu \mathrm{g} \mathrm{L}^{-1}$ : cachaça $\left(0,109 \mu \mathrm{g} \mathrm{L}^{-1}\right)$, rum $\left(1,33 \times 10^{-2} \mu \mathrm{g}\right.$ $\left.\mathrm{L}^{-1}\right)$ e uísque $\left(4,85 \times 10^{-2} \mu \mathrm{g} \mathrm{L} \mathrm{L}^{-1}\right)$.

De nosso conhecimento, não existe menção na literatura sobre a presença de HPAs em etanol utilizado como combustível. No entanto, é de se esperar que sua origem ocorra de forma semelhante ao verificado para a cachaça e o rum. ${ }^{5,6,9,10}$

Os valores de concentração (Tabela 1S) para os 15 HPAs nas 51 amostras de bebidas (14 cachaças, 19 rum e 18 uísque) foram analisados por $P L S-D A$ e organizados em uma matriz $15 \times 51$, na qual as concentrações foram normalizadas e depois submetidas à análise multivariada (quimiometria). A análise quimiométrica indicou os seguintes hidrocarbonetos como descritores químicos: F, FE, AN, FL, PI, CR, BaP, DBahA e BghiP.

$\mathrm{Na}$ análise por $P L S-D A$, as variáveis foram escolhidas por meio da exclusão dos analitos que apresentaram os menores valores nas componentes 1 e 2 . Uma inspeção no gráfico de loading (Figura 3) mostra que a componente $1(32,0 \%)$ está relacionada com os teores de PI, FE, BaP e F, enquanto que a componente 2 (19,2\%) está relacionada principalmente com os teores de DBahA, AN, BghiP, CR e FL.

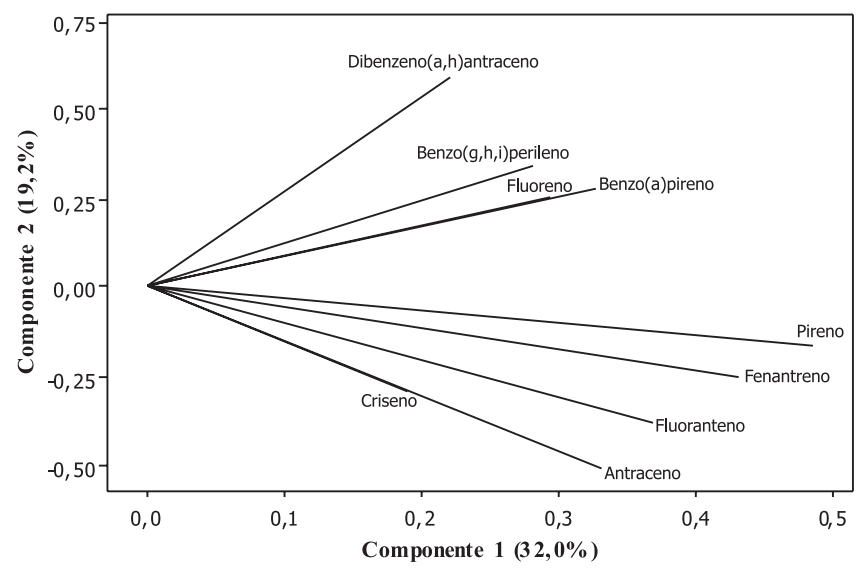

Figura 3. Gráfico de loading da PLS-DA
A Figura 4 reflete a tendência observada para as concentrações médias de HPAs (Tabela 1), as quais aumentaram na sequência: rum, uísque e cachaça, formando três aglomerados distintos. O gráfico de scores (Figura 4) mostra uma tendência das amostras de bebidas destiladas se dispersarem ao longo do eixo da componente 1. Nesta figura é possível notar a formação de três grupos distintos, melhor visualizados pelas elipses arbitrariamente delimitadas para cada tipo de bebida. Verifica-se também na Figura 4 que das 18 amostras de uísques, apenas 2 (uísques 12 e 13) não estão devidamente situadas na região definida pelas demais amostras, isto em função dos teores mais baixos de PI e CR.

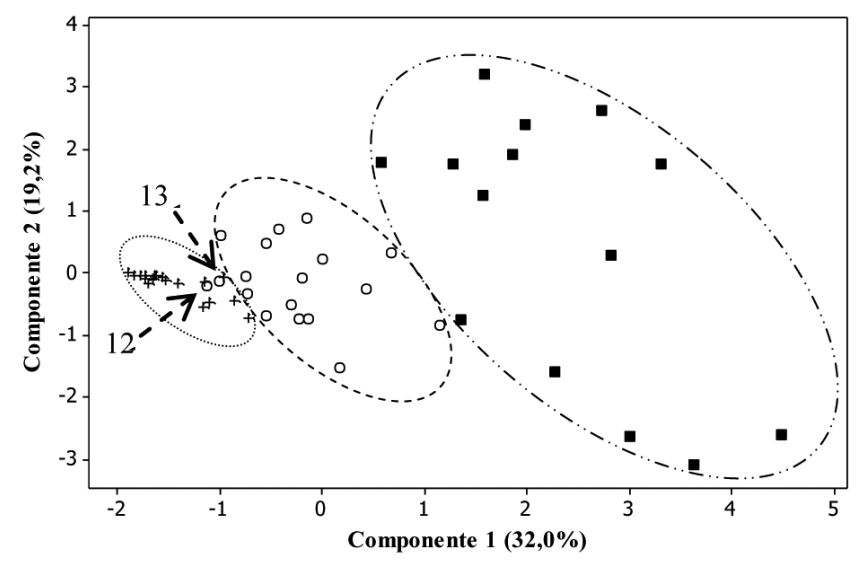

Figura 4. Gráfico de scores da PLS-DA das amostras de rum (+), uísque (O) e cachaça (

As duas componentes do gráfico de scores (Figura 4) apresentaram uma explicação do banco de dados original de $51,2 \%$, indicando que os destilados podem ser diferenciados quanto a sua contaminação por HPAs.

Utilizando-se o modelo quimiométrico desenvolvido ${ }^{15}$ para cachaça empregando-se a técnica de $L D A$, procurou-se verificar a origem destes derivados de cana-de-açúcar com respeito ao tipo de cana utilizado em sua produção (queimada ou não queimada), utilizando-se AN, AC, F, FE, AN, FL, PI, BaA, BbF e BghiP como descritores químicos. ${ }^{15} \mathrm{O}$ modelo desenvolvido anteriormente ${ }^{15} \mathrm{e}$ a sua validação cruzada (matriz 15 x 103) apresentam uma porcentagem total de acerto de 98,1 e $95,1 \%$, respectivamente.

A sua aplicação para as 39 amostras de derivados de cana-deaçúcar (14 cachaças, 19 rum e 6 álcool combustível) indicaram que 3 das 14 amostras de cachaça testadas (3, 5 e 6 da Tabela 1S) se enquadram no grupo das amostras produzidas com cana de açúcar queimada. O mesmo se aplica a 2 das 6 amostras de álcool combustível (etanol 4 e 5). Todas as 19 amostras de rum apresentaram características semelhantes às amostras produzidas com cana não queimada.

\section{CONCLUSÕES}

Os resultados analíticos confirmaram a presença de HPAs em cachaça, rum, uísque e álcool combustível. Observou-se que o naftaleno e o fenantreno são os HPAs mais abundantes nas amostras de cachaça e rum. Já para o uísque, foram o naftaleno e o fluoranteno. Para o álcool combustível, os hidrocarbonetos mais abundantes foram o antraceno e o fluoranteno.

A soma dos teores médios de HPAs decresce na ordem: cachaça (9,43 $\left.\mu \mathrm{g} \mathrm{L}^{-1}\right)$, uísque $\left(7,30 \mu \mathrm{g} \mathrm{L}{ }^{-1}\right)$, álcool combustível $\left(5,70 \mu \mathrm{g} \mathrm{L}^{-1}\right)$ e rum $\left(1,98 \mu \mathrm{g} \mathrm{L^{-1 }}\right)$.

A aplicação do modelo, ${ }^{15}$ desenvolvido para amostras de cachaça, utilizando a técnica de $L D A$, indica que 5 amostras de derivados de 
cana-de-açúcar ( 3 de cachaça e 2 de álcool combustível) foram produzidas com cana queimada, e que as amostras de rum se enquadram na categoria de bebidas produzidas com cana não queimada.

\section{MATERIAL SUPLEMENTAR}

A Tabela $1 \mathrm{~S}$, referente à concentração de HPAs nas amostras de cachaça, rum, uísque e álcool combustível (etanol), encontra-se disponível gratuitamente em http://quimicanova.sbq.org.br na forma de arquivo PDF.

\section{AGRADECIMENTOS}

Ao CNPq, à CAPES e à FAPESP pelo apoio financeiro.

\section{REFERÊNCIAS}

1. Arrebola, F. J.; Frenich, A. G.; Rodrígues, M. J. G.; Bolaños, P. P.; Vidal, L. M.; J. Mass Spectrom. 2006, 41, 822.

2. Pagliuca, G.; Gazzotti, T.; Zironi, E.; Serrazanetti, G. P.; Mollica, D.; Rosmini, R.; J. Agric. Food Chem. 2003, 51, 5111.

3. Netto, A. D. N.; Moreira, J. C.; Dias, A. E. X. O.; Arbilla, G.; Ferreira, L. F. V.; Oliveira, A. S.; Barek, J.; Quim. Nova. 2000, 23, 765.

4. EUROPA, Commission Regulation (EC) $\mathrm{n}^{\circ}$ 1881/2006, of 19 December 2006, Official Journal of the European Union, of 20/12/2006.

5. Magalhães, D.; Bruns, R. E.; Vasconcelos, P. C.; Quim. Nova. 2007, 30, 577.

6. Galinaro, C. A.; Dissertação de Mestrado, Universidade de São Paulo, Brasil, 2006.

7. Moret, S.; Amici, S; Bortolomeazzi, R.; Lercker, G.; LebensmittelUntersuchung und Forschung. 1995, 201, 322.
8. Kleinjans, J. C. S.; Moonem, E. J. C.; Dallinga, J. W.; Albering, H. J.; Van Den Bogaard, A. E. J. M.; Van Schooten, F. J.; The Lancet 1996, 348,1731

9. Galinaro, C. A.; Cardoso, D. R.; Franco, D. W.; J. Agric. Food Chem. 2007, 55, 3141.

10. Tfouni, S. A. V.; Machado, R. M. D.; Camargo, M. C. R.; Vitorino, S. H. P.; Vicente, E.; Toledo, M. C. F.; Food Chem. 2007, 101, 334.

11. Mosedale, J. R.; Puech, J. L.; Int. J. Food Sci. Technol. 1998, 9, 95.

12. Patrício, I.; Canas, S.; Belchior, A. P.; Ciênc. Téc. Vitiv. 2005, $20,1$.

13. Cardoso, D. R.; Andrade-Sobrinho, L. G.; Leite-Neto, A. F.; Reche, R. V.; Isique, W.D.; Ferreira, M. M. C.; Lima-Neto, B. S.; Franco, D. W.; J. Agric. Food Chem. 2004, 52, 3429.

14. Bruns, R. E.; Faigle, J. F. G.; Quim. Nova. 1985, 8, 84.

15. Galinaro. C. A.; Cardoso, D. R.; Franco, D. W.; BR PI 0.705.458-0, 2007.

16. Sampaio, O. M.; Reche, R. V.; Franco, D. W.; J. Agric. Food Chem. 2008, 56,1661

17. Sivertsen, H. K.; Holen, B.; Nicolaysen, F.; Risvik, E.; J. Sci. Food Agric. 1999, 79, 107.

18. Dr Luis O. Gálvez Taupier (ICIDCA-Cuba), comunicação pessoal.

19. García-Falcón, M. S.; Simal-Gándara, J.; J. Food Add. Contam. 2005, $22,791$.

20. Moret, S.; Conte, L. S.; J. Chromatogr., A 2000, 882, 245.

21. Pagliuca, G.; Gazzotti, T.; Zironi, E.; Serrazanetti, G. P.; Mollica, D.; Rosmini, R.; J. Agric. Food Chem. 2003, 51, 5111.

22. BRASIL, $R D C \mathrm{n}^{\circ} 274$, de 22 de setembro de 2005, Diário Oficial da União, de 23/09/2005.

23. BRASIL, $R D C \mathrm{n}^{\circ} 2$, de 15 de janeiro de 2007, Diário Oficial de União, de $17 / 01 / 2007$. 


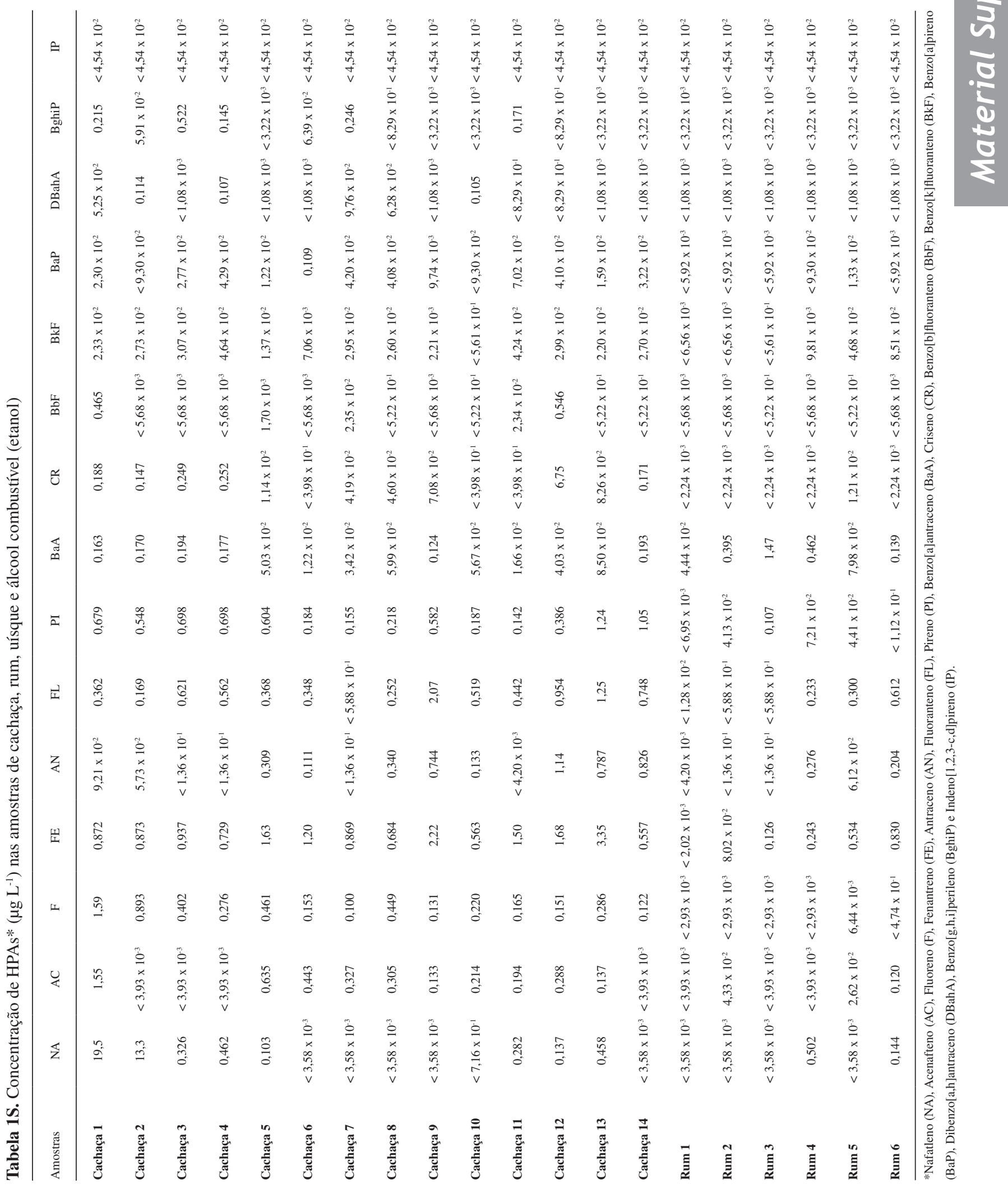




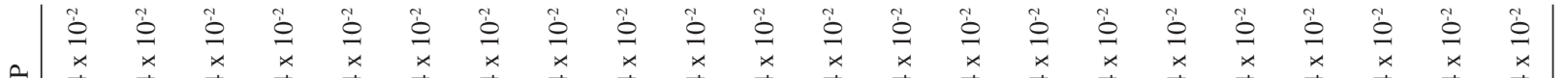

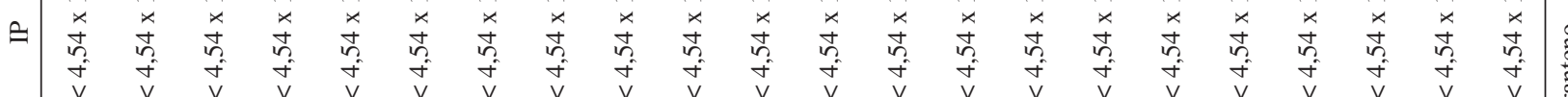

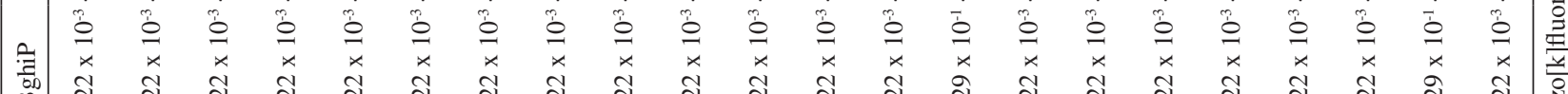

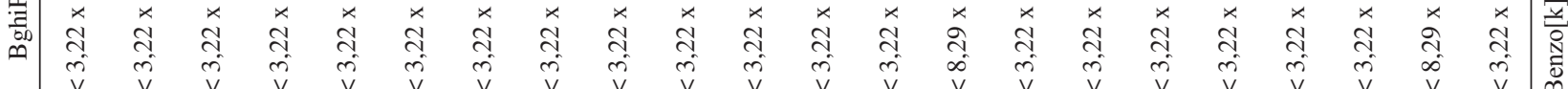

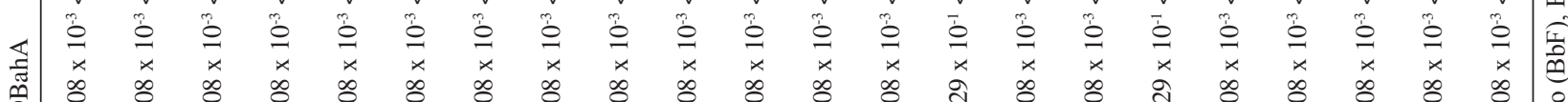

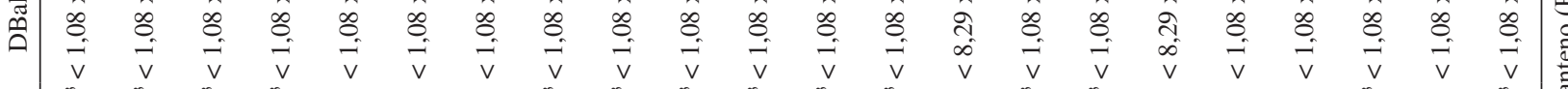

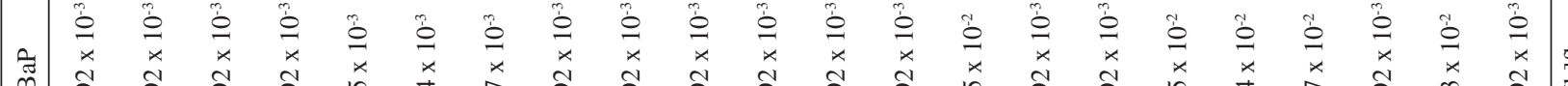

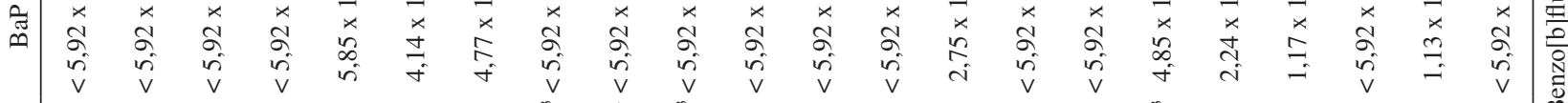

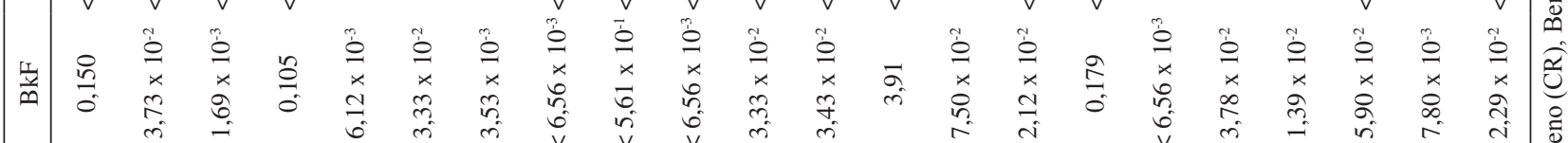

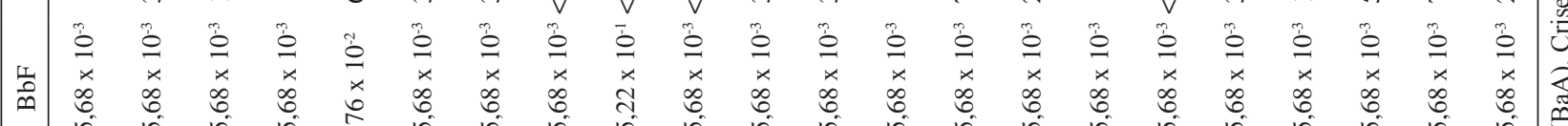

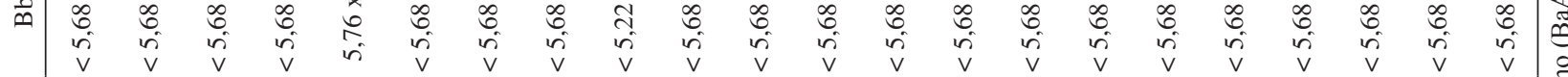

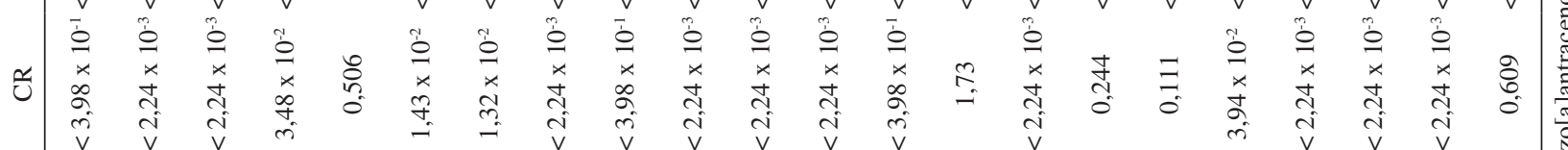

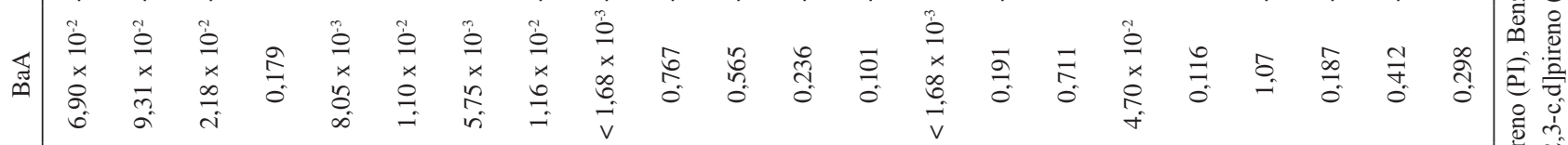

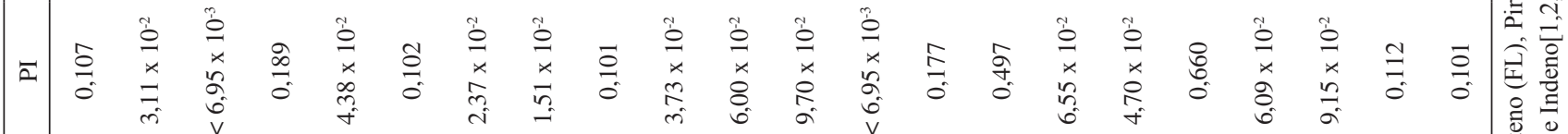

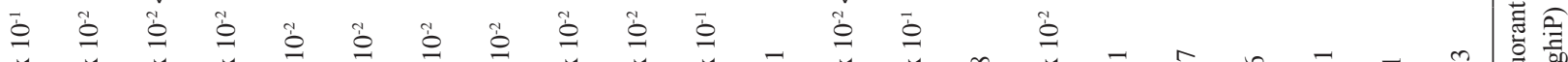

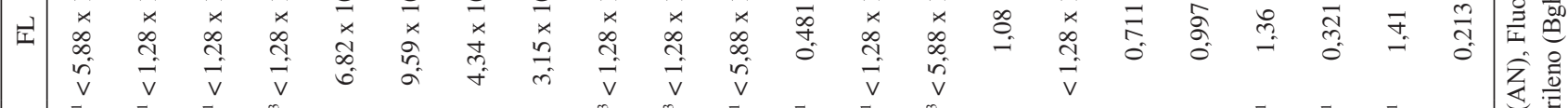

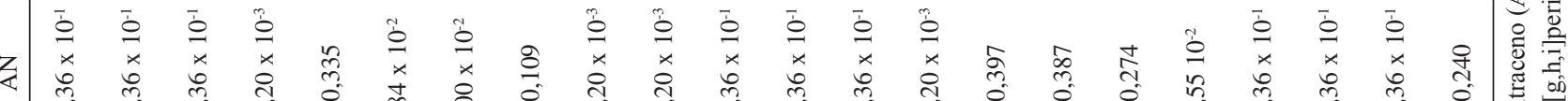

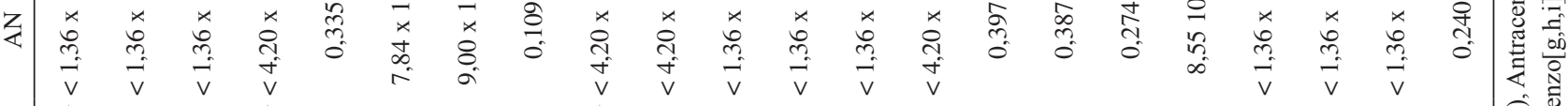

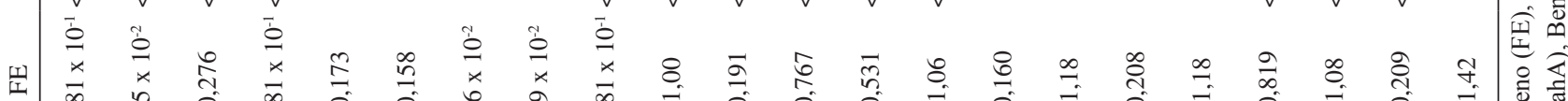

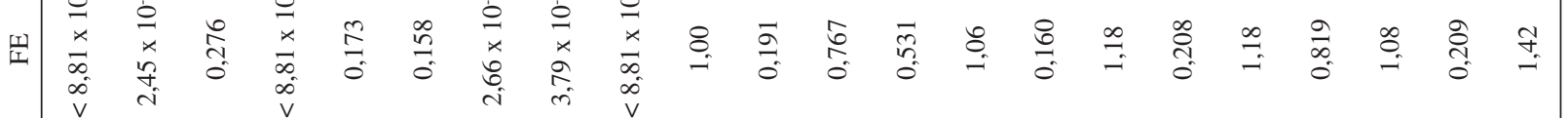

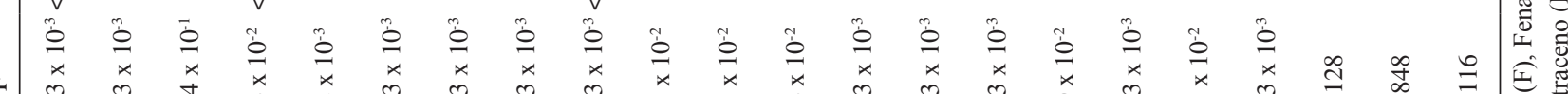

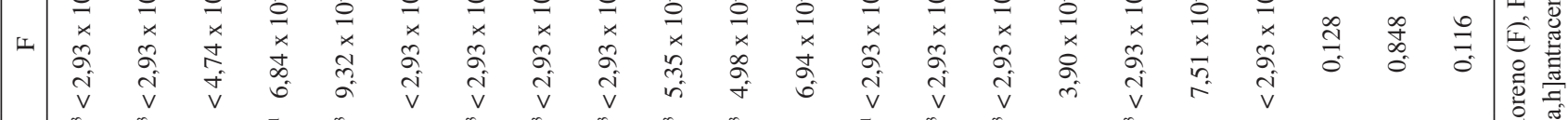

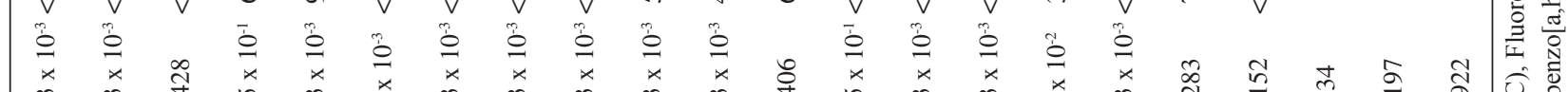

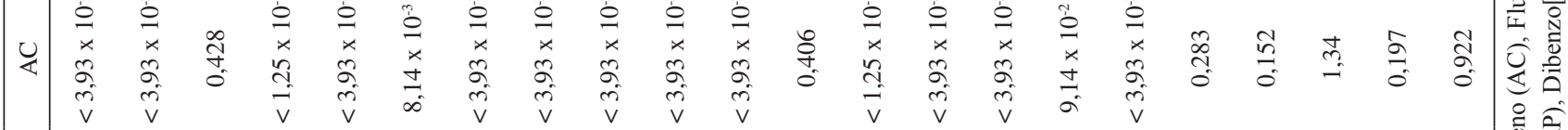

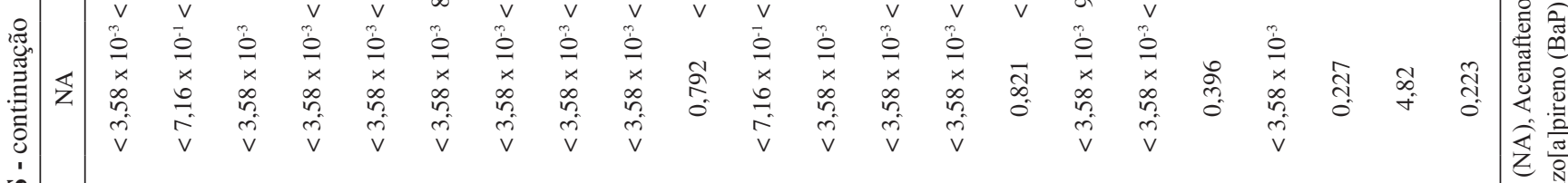

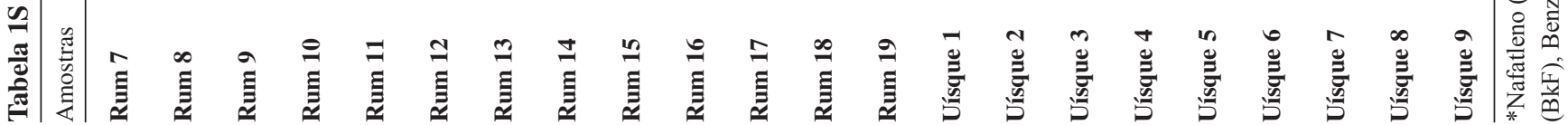




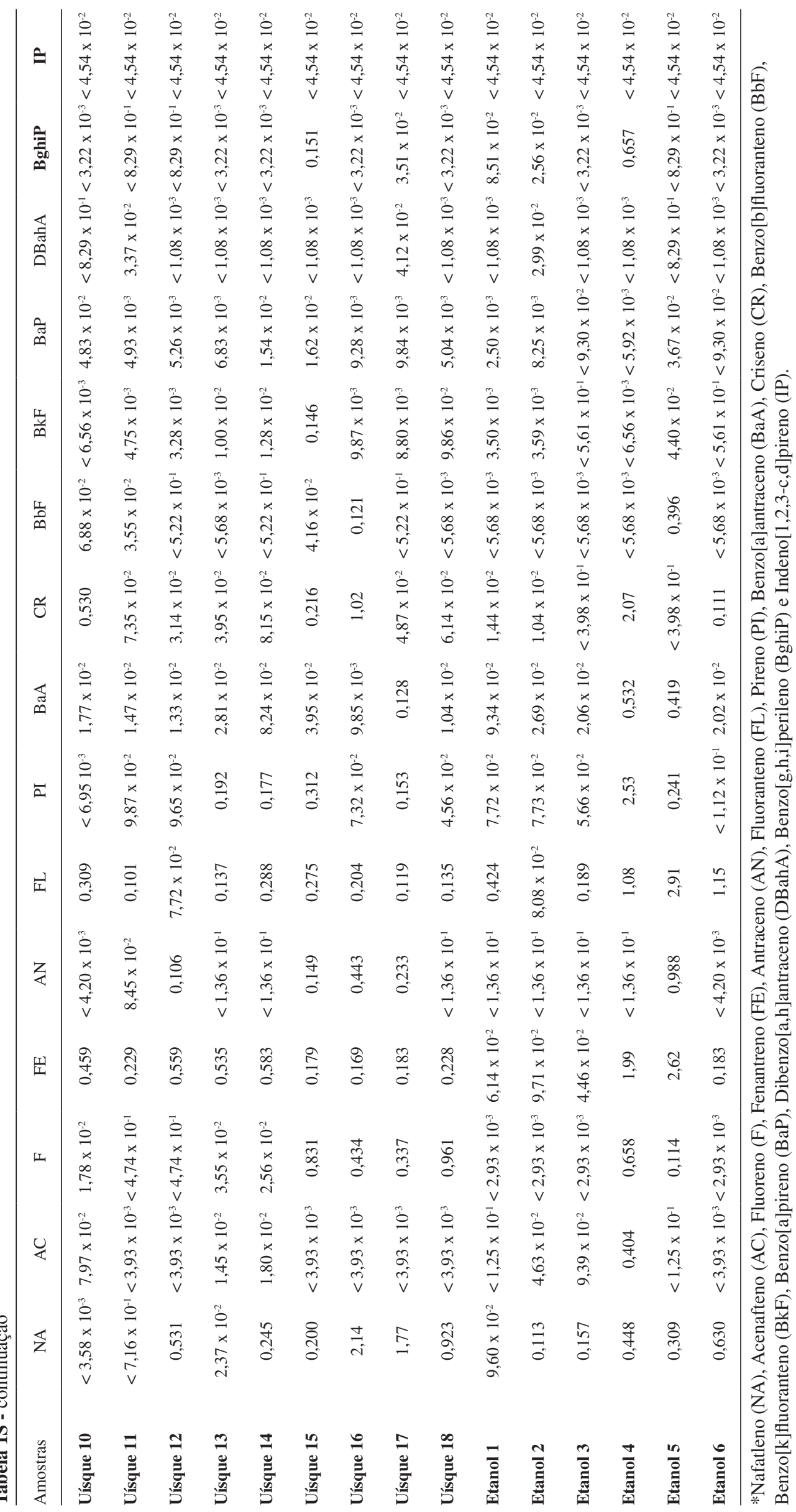

